\title{
Game-based Crisis Simulation and Generation Framework: Design and Implementation Structure
}

\author{
Pisit Praiwattana and Abdennour El Rhalibi \\ Department of Computer Science \\ Liverpool John Moores University, \\ Byrom Street L3 3AF, Liverpool UK \\ P.Praiwattana@2014.ljmu.ac.uk, a.elrhalibi@ljmu.ac.uk
}

\begin{abstract}
Crisis is an infrequent and unpredictable event. Training and preparation process requires tools for representation of crisis context. Particularly, Crisis Event consists of different situations which can occur at the same time combining into complex situation and becoming a challenge in collaboration of several crisis management departments. Studying of Resource distribution also improving an effectively in solving the ongoing crisis. By integrating modern game technology, development process of assistance and simulation system can become a cost-effective solution to allow observation and test practice procedures. Therefore, we aim to discuss and provide an implementation design choices of general framework tool for representing of coverage terrain, resources, different stakeholders and structure of crisis scenario using Unity3D game engine technology. The paper focuses on the procedural generation of complex 3D environment for crisis scenarios generation and disaster management, and introduces the framework, structure, functions and the visualization, and performance evaluation of the framework.
\end{abstract}

Keywords: Crisis Simulation Framework, Procedural Computer Generation, Agent-Based System

\section{Introduction}

Currently, an occurrence of changing in world-environment states is commonly fast-paced and unpredictable. Such situation which may result in large scale of adversary impact on environment, population and resources can be considered as crisis situation. In preparation stage, the practices of distributing manpower, setting up tools, considering time expense are essentially required to derive an effective plan for complex situations. Fortunately, game-based application provides a cost-effective solution, as a game development technology such as Unreal Engine and Unity3D have been currently more accessible for researchers, and, thus, it becomes an affordable tool for integrating the visual representation and simulation process of emerging disaster to develop a practice application for crisis personal. 
By representing and simulating ongoing crisis situation into description of scenario-based structure, it allows finding an answer to a question of what is the optimal responses in allocating, deploying, and prioritizing of available resource parameters and operation protocols. Regarding of the scope in crisis scenario representation, models can be designed using a level of information perception and possible interaction of participants. For instance, it can be designed with role-playing approach in first-person perspective on limited local perception of event, or it can have been further extended into real-time strategy observation approach in top-view with filtered information on resources and multiple events. Although both example models are aiming to reproduce a training experience in executing official procedures, the former design is suitable for training specific scope of crisis operational agents while the latter is often more appropriated for observing and testing large scale deployment plan on time-contesting situation. These design decisions strongly influence on how the final application will be deployed and evaluated.

In this paper, we provide a brief discussion on the existing scenario generation system then identify the core features of crisis simulation framework. Further, we discuss on general design decision and implementation of proposed framework in details. The remainder of this paper is organized as follows: In Section 2, we introduce backgrounds on automated content generation system and example of crisis simulation systems; In Section 3, we discuss implementation design of each component for our proposed framework; In Section 4, we discusses the result and evaluation of visualization stage; and, in section 5 , we conclude the paper.

\section{Backgrounds}

\subsection{Automated Content Generation System}

Modern serious games will required scenario and visual asset to provide content. There are also research which focus on procedural generation of such content.

Related researchers have tackled procedurally generated game environments with varying degrees of success. The most well known and most significant research has been carried out on the CityEngine [1], a system that is capable of producing realistic and detailed models. The generation algorithms are inspired by the modeling of natural phenomena in string grammars [2] and L-systems are used to construct road networks and buildings [1]. Procedural building generation has focused on the application of grammars to describe structure, like the Shape Grammars original proposed by [3]. These have been applied in various guises to the construction of building geometry [4].

In [5], the authors define a specific grammar to characterize building structure. This system defines rules to operate on shapes and can be used to design a range of detailed buildings in several architectural styles. Recently this approach has been extended to employ imaging techniques to aid in the acquisition of generation rules from existing building facades $[6,7]$.

Other approaches include the application of intelligent agents [8], real-time frustum filling [9] and template based generation [10]. In $[11,8]$ the authors 
propose to simulate the evolution of cities by modelling land use and evolving a city usage map over time that can be used later to create a cityscape. Real-time city generation has also been attempted.

In $[9,11]$ the authors implemented a city generation system that is filling the view frustum rapidly with buildings of various shape combinations but their placement have been restricted to a road network consisting of a regular grid. In [10] the authors proposed an application of templates that encapsulate patterns such as raster radial to generate road networks.

Compared to these techniques, our approach introduces several novel aspects including: use of agent-based modeling for the game world generation, interoperability solution development using Unity $3 \mathrm{D}$ and allowing deployment across many platforms including web browsers, and multiple OS; instant in-game generation, rendering and recreation of 3D hexagonal cell-based and organic environments; agent AI Bots to interact with the environment after generation stage and according to other user-set constraints, and structural constraints induced by the game world and crisis scenario generation; and full control of the environment editing by the user.

\subsection{Crisis Simulation Systems}

There are several research of proposed crisis simulation system focusing on variety of implementation scopes which some have been built for specific platform and application. In general, the main objective of developing such a system is to provide practice experience on prepared protocols for related personals based on occurring crisis event. The deployment of simulation system may be integrated in complete game-based application, virtual equipment training, or combining real-system with content-simulation module. Furthermore, in simulating of realworld disasters on constrained situation of environments and stakeholders such as epidemic outbreak, wildfire and flood, deploying Agent-based model (ABS) architecture will provide ease of observance and flexibility to assign behaviors for appropriate entities. $[12,13]$.

For example, a multi-agent system WIPER [14] is using real GIS data for visualizing geographic terrain while each mobile phone user is having a movement activity tracked by tower cellular segments covering the area for a real-time response. The system allows observance of variety in movement and evacuation pattern with traffic situation. In SimGenis, it is proposed to design optimal, efficient, and appropriate rescue strategies, based on the initial state of victims, number of rescuers, and method of communication between rescuers. More precisely, the original aim of their research is determining how to response a dynamic large-scale emergency depends on the use of a centralized and decentralized collaborative rescue strategy with applying heuristic algorithm on each agent and component in simulator [15]. Robocup Rescue [16,17] which used the 1995 Kobe earthquake as the original test scenario. The system is aiming to represent the disaster situation sensory information then to incorporate the agent-simulation system to mitigate disaster and encourage large scale research collaboration based on optimization of the design and implementation of better 
action selection method. Moreover, PLAN-C $[18,19]$ is also another ABS developed to predict the behavior of individual and collectively organized agents in large-scale emergency such as terrorist attack. Lastly, EpiSimS [20,21] is also another ABS developed to study the optimized parameters of resource and procedures in a SmallPox scenario model and influenza outbreak including the behavior of infected patients with deployment pattern of related officials. From our survey [22], crisis simulation systems shared similarities in: (1) abstracting the representation of real-world locations, participated stakeholders and environment entities; (2) reproducing crisis scenario based on knowledge and historical records; and, ultimately, (3) to train a crisis personal with appropriate response for any upcoming situations. The simulation stage also being benefit from having integrated automated content generation to tailor progression of crisis situations due to the nature of large scale disaster being very complex and restrained the time of crisis scenario designer.

In summary, these researches provided insight on possible core components of general crisis simulation framework which are representation and generation of terrain; process of distributing and storing key resource; procedures for facilities and specialized crisis agents in deployment, communication and collaboration; simulation of crisis event and integrating agent-based model to replicate responses of impacted stakeholders which can be individual citizens, responsible governing unit, specific department and personal using planning or rule-based approach; and finally integration of automated content generation on both terrain visualization and crisis scenario. We will propose and discuss the implemented of framework which aims to address the overall structure of these core components in the next section.

\section{Crisis Scenario Simulation and Generation Framework}

The crisis scenario simulation framework is separated into 2 focus groups of core components. The former is to consider representation structure of area, resources and stakeholder in the situation. Next, the latter is dealing with simulation of behaviors from these stakeholders respectively to their role and authority, while the crisis scenario generation will based on simulation framework to deliver a combination of these representations and tailoring sequence of event and situations logically or from the prescript of conditions. This section will only focus on initial setup of crisis simulation framework only. The uniqueness of application in proposed structure is that it adopts the variety of representation and simulation whether it is game-based model like SimCity or limited resource management situation on specific crisis context. Figure 1 shows the framework architecture with core components and figure 2 displays the implemented result in Unity3D. 


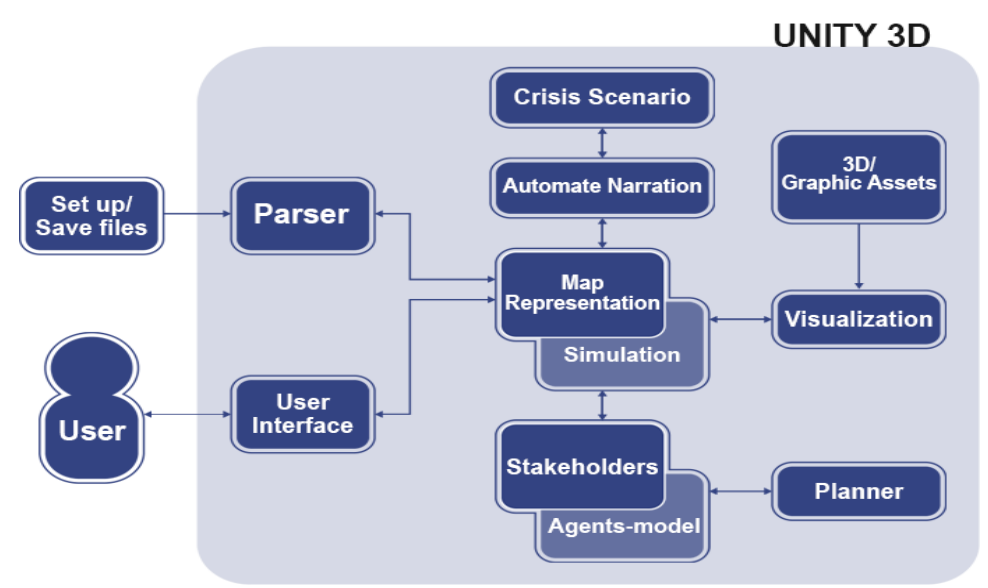

Fig. 1: Framework architecture with core components

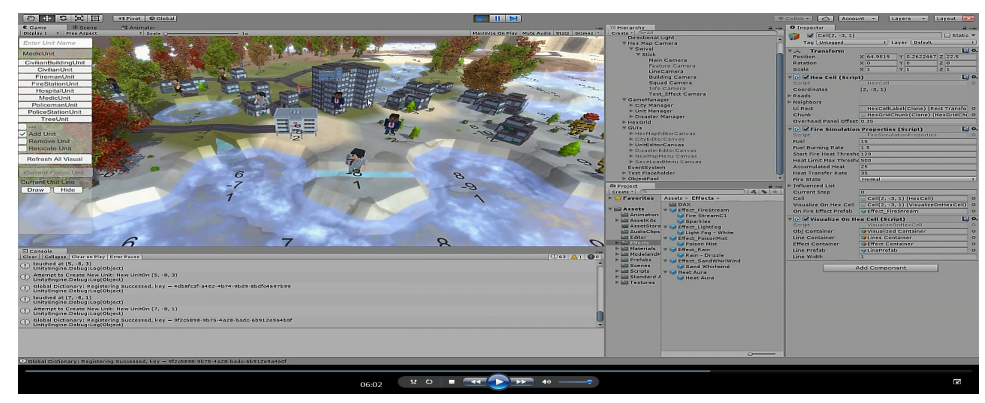

Fig. 2: Framework architecture in Unity 3D

\subsection{Map Representation}

The map space for proposed framework is represented as abstract grid of hexagon cell (Hex Cell) which consists of 6 cell neighbors locating in cube coordinates on Cell(X, Y, Z). The distinct advantage from choosing hex cell over the traditional square cell lattice is that the distance between each direction to center of the cell would be symmetrical and, therefore, provide more realistic representation in traversing between coordinates. Each cell stored information of its terrain component type, elevation unit, resource supply being stocked, and other flags for determining the information of details such as road, river and etc. Necessary parameters for determining simulation event such as temperature or water mass can be added on specific cell individually.

\subsection{Stakeholders}

The stakeholders in crisis situation are being classified into three different representations accordingly; (1) City; (2) Facility; and (3) Personal Unit. 
City is representing a governing unit over a set of specific cell areas and has one of its cells representing a city center. As a representative of governing unit, city holds authority over central collective resources from its coverage area with high-level information of crisis event and resources. City makes decision on resources and unit assignment.

Facility is representing the base of operation for specific department residing in one area cell. It includes a temporary base of operation, evacuation area, and storehouse. These facility-type stakeholders are often being assigned to hold a resource supply to execute action. Each facility is having a local view of situation based on its operational areas. Some equipping with agent-based behavior system will assess ongoing event associating with provided role of responsibility based on the remaining resources then decide to execute logical action such as dispatching crisis agent units equipped with required level of resources to solve the situation or requesting for replenishment of supply when the storage is considered to be not sufficient or depleted.

Personal Unit (or Unit) is, in general, a representation of quantity in mobile group of individual. There are two basic distinct roles of unit as a crisis personal squad and civilian. The former, squad is holding a responsibility role respectively such as Firemen, Medical doctors, Policemen and being assigned a quantity of resource supply from its facility owner for executing direct solving action on the crisis events

The latter, civilians is representing represented in the situation such as injuries injured and escaping individuals from the impact area. These personal units have a low-level information perspective to only its specific role objective and, thus, will execute a fundamental action to reach the goal condition.

\subsection{Crisis Scenario}

To providing a basic structure, crisis scenario context will be described as a set of Crisis Events. For each event, it is also consisting of another set of Disaster Situations and starting time in the simulation clock called Simulation Step in which one step is including calculation of ongoing disaster flag such as fire and flood with evaluation of simulation parameters for state-changing.

Disaster Situation is considered as template for any anomaly of raise or decrease of simulation parameters similar to real-world problem. This allows us to describe the element of situation specifically in parameters and being reusable for similar context. For an example, the Heat surge situation would raise the temperature parameter of specific area with 30-50 degrees and, therefore, allowing Fire Simulation Properties to trigger a burning state if the condition is reaching the starting fire temperature threshold.

Crisis Event is considered to be an abstracted event combining different situations altogether. The event will consist of delay in simulation step which control the timing to be active state. Next, activated crisis event is to applying different disaster situations on designated cells. From the representation of crisis event, it allows us to represent a single event as a specific template which is reusable similar to disaster situations. 
Lastly, Crisis Scenario is a high-level narrative of sequenced crisis events. With the collective representation of crisis events and disaster situations, the specific scenario can be reproduced with flexibility of alteration such as a random starting time of crisis event and range of disaster situation impact on parameter scale modifier.

\subsection{Visualization}

Fundamentally, Grid-based data structure allows us to represent the positioning of single strategic area space as cell and concept of residing resource, facilities, personals, and state of properties clearly. Therefore, visualization component is processing the map grid then, for each cell, calculates the vertices for terrain mesh triangulation from their coordinates and elevation. The quantity of resource supply is parsed into level of visual feature parameters for flexibility in 3D Assets preparation. Next, the placement of higher level abstraction will be facilities locating in the cell alongside with personal unit similar to visual representation of real-time strategy game. This similarity provides ease of perception using common game-metaphor and fast recognition to the user. As situation on each cell is varied based on its setup but, in general, it is to select a correct visual effect determining for specific situation. Optimization is preliminary done by using Built-in Unity3d Level-of-Detail object which switch preassigned different details of model or sprites based on camera distance.

\subsection{Simulation of Environment}

The simulation control will hold a local clock which is mentioned in section 3.3 as simulation step. This step represents one loop to applying disaster situation parameters to associated components; evaluating the components state; and simulating the impact of state such as transferring heat to nearby area, depleting fuel or flow the exceeding water mass respectively; applying damage value to residing personal units in the affected area. Lastly, adjust the visual effect or cell parameters as final result. The algorithm for example fire and flood disaster situation is initially designed by applying basic of a cellular automaton behavior considering the influence of nearby cells neighbors parameters to calculate and change internal state of each cell accordingly.

\section{Result and Performance Evaluation}

Crisis scenario simulation and generation framework has been implementing on Unity3d Game Engine for ease of visualization process. This section explains a result on process of terrain generation and visualization. The framework experiments was run in a Laptop with Core i7-5500U @2.40 GHz, 2 Cores with RAM 16GB (10GB Available), and Window 10 with GeForce 840m model. Figure 3 shows the overall visualized results from parsing terrain information on close-up detail and zoomed images on different size of terrain setups, (a) Small map with 
grid size of $25^{*} 15$ of total 300 cells; (b) Medium map with grid size of $40^{*} 30$ of total 1200 cells; (c) Large map with grid size of $80 * 70$ of total 5600 cells.

The overall visualization performance of proposed framework yielded general desirable for game application in small to medium map setup and drop to half framer-ate in large map setup. Terrain and water surface visualization were functioning on above average of $60 \mathrm{fps}$. Turning on the decoration map features resulted in linear decrease of framerate while units placement and visual effect rendering did not greatly slow down the system. In close-up scene view, size of the terrain map also represented linear decrease of framerate. Although small and medium map can still provide functional framerate during runtime, the large map size have suffered drop in framerate significantly to an average of $15 \mathrm{fps}$, when turning on visualization of scene features.

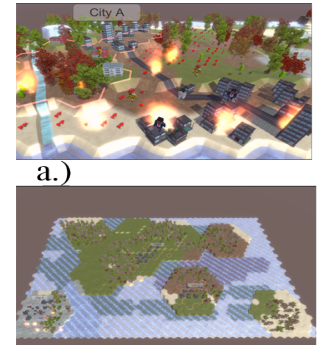

c.)

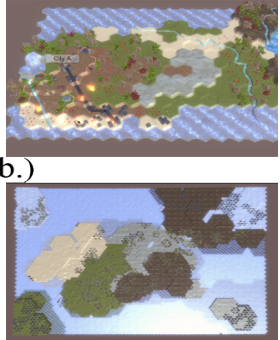

d.)

Fig. 3: Result for a.) Close-up; b.) Small map c.) Medium map (c) Large map

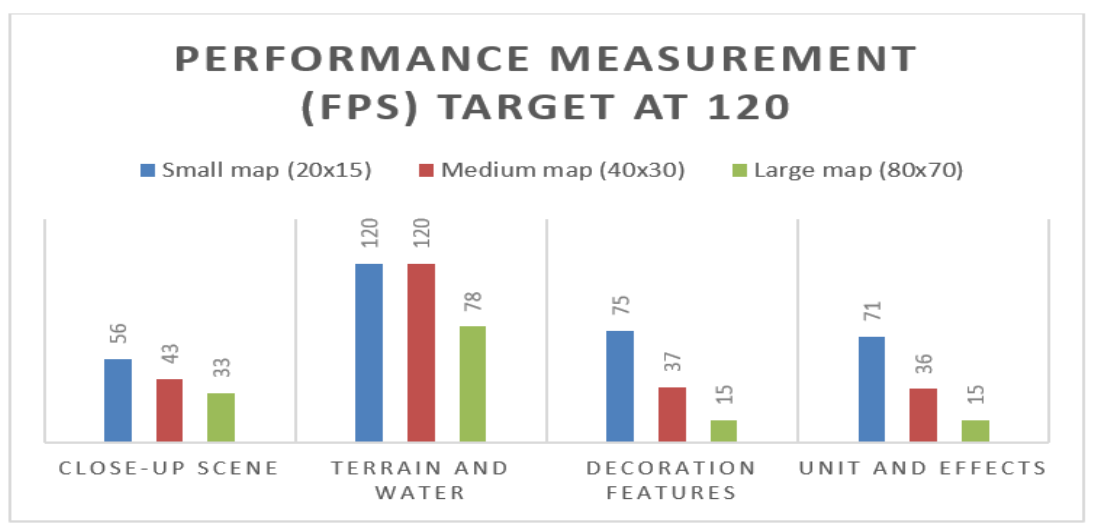

Fig. 4: Performance measurement in frame per seconds at target limit of 120 frames on a.) Close-up scene with all options on b.) Terrain and water only c.) Decoration features and 3D models d.) Placement of units and visual effects 


\section{Conclusion}

This paper discusses the core components of proposed Crisis Scenario Simulation and Generation framework including its structure for representing terrain area, stakeholders, crisis scenario and visualization process.

The provided implementation result on representation of core component and testing in visualization stage from our framework shows that it is a flexible tool to support modeling, visualization and testing of general crisis scenario situation. Implemented on Unity3D game engine, the game-based application will be benefit from porting into different platform including web-based, providing accessibility to crisis scenario manager, designer, crisis personals and interested individuals. This work is preliminary, and whilst the planning system has been developed, the integration with visualization system and crisis generation scenario and simulation is on-going.

In the future work, our effort will focus on integration and evaluation of the agent-based model with planning capabilities to deliver a crisis plan with optimal actions.

\section{References}

1. Bekins, D., Aliaga, D.G.: Build-by-number: Rearranging the real world to visualize novel architectural spaces. In: Visualization, 2005. VIS 05. IEEE. pp. 143-150. IEEE (2005)

2. Greuter, S., Parker, J., Stewart, N., Leach, G.: Real-time procedural generation of 'pseudo infinite' cities. In: Proceedings of the 1st International Conference on Computer Graphics and Interactive Techniques in Australasia and South East Asia. pp. 87-ff. GRAPHITE '03, ACM, New York, NY, USA (2003), http://doi. acm.org/10.1145/604471.604490

3. Lechner, T., W.B.W.U., Felsen, M.: Procedural city modelling. Tech. rep., Northwestern University, November (2003)

4. Lechner, T., W.B.R.P.W.U.T.S., Felsen: Procedural modelling of land use in cities. Tech. rep., Northwestern University, August (2004)

5. Müller, P., Wonka, P., Haegler, S., Ulmer, A., Van Gool, L.: Procedural modeling of buildings. In: ACM SIGGRAPH 2006 Papers. pp. 614-623. SIGGRAPH '06, ACM, New York, NY, USA (2006), http://doi.acm.org/10.1145/1179352.1141931

6. Müller, P., Zeng, G., Wonka, P., Van Gool, L.: Image-based procedural modeling of facades. In: ACM SIGGRAPH 2007 Papers. SIGGRAPH '07, ACM, New York, NY, USA (2007), http://doi.acm.org/10.1145/1275808.1276484

7. Parish, Y.I.H., Müller, P.: Procedural modeling of cities. In: Proceedings of the 28th Annual Conference on Computer Graphics and Interactive Techniques. pp. 301-308. SIGGRAPH '01, ACM, New York, NY, USA (2001), http://doi.acm. org/10.1145/383259.383292

8. Prusinkiewicz, P., Lindenmayer, A.: Modeling of cellular layers, pp. 145174. Springer New York, New York, NY (1990), http://dx.doi.org/10.1007/ 978-1-4613-8476-2_7

9. Stiny, G.: Introduction to shape and shape grammars. Environment and Planning B: Planning and Design 7(3), 343-351 (1980), http://dx.doi.org/10.1068/ b070343 
10. Sun, J., Yu, X., Baciu, G., Green, M.: Template-based generation of road networks for virtual city modeling. In: Proceedings of the ACM Symposium on Virtual Reality Software and Technology. pp. 33-40. VRST '02, ACM, New York, NY, USA (2002), http://doi.acm.org/10.1145/585740.585747

11. Wonka, P., Wimmer, M., Sillion, F., Ribarsky, W.: Instant architecture. ACM Trans. Graph. 22(3), 669-677 (Jul 2003), http://doi.acm.org/10.1145/882262. 882324

12. Wooldridge, M., Jennings, N.R.: Intelligent agents: Theory and practice. The knowledge engineering review 10(02), 115-152 (1995)

13. Challenger, R., Clegg, C., Robinson, M., Leigh, M.: Understanding crowd behaviours: Simulation tools. UK Cabinet Office (2009)

14. Schoenharl, T., Madey, G.: Design and implementation of an agent-based simulation for emergency response and crisis management. Journal of Algorithms \& Computational Technology 5(4), 601-622 (2011)

15. Saoud, N.B.B., Mena, T.B., Dugdale, J., Pavard, B., Ahmed, M.B.: Assessing large scale emergency rescue plans: an agent based approach. The International Journal of Intelligent Control and Systems 11(4), 260-271 (2006)

16. Takeuchi, I.: A massively multi-agent simulation system for disaster mitigation. In: International Workshop on Massively Multiagent Systems. pp. 269-282. Springer (2004)

17. Siddhartha, H., Sarika, R., Karlapalem, K.: Score vector: A new evaluation scheme for robocup rescue simuation competition 2009. Rescue Technical Committee (2009)

18. Mysore, V., Narzisi, G., Nelson, L., Rekow, D., Triola, M., Shapiro, A., Coleman, C., Gill, O., Daruwala, R.S., Mishra, B.: Agent modeling of a sarin attack in manhattan. In: Proceedings of the First International Workshop on Agent Technology for Disaster Management, ATDM. pp. 108-115 (2006)

19. Narzisi, G., Mysore, V., Mishra, B.: Multi-objective evolutionary optimization of agent-based models: An application to emergency response planning. Computational Intelligence 2006, 224-230 (2006)

20. Barrett, C.L., Eubank, S.G., Smith, J.P.: If smallpox strikes portland... Scientific American 292(3), 54-61 (2005)

21. Mniszewski, S.M., Del Valle, S.Y., Stroud, P.D., Riese, J.M., Sydoriak, S.J.: Episims simulation of a multi-component strategy for pandemic influenza. In: Proceedings of the 2008 Spring simulation multiconference. pp. 556-563. Society for Computer Simulation International (2008)

22. Praiwattana, P., El Rhalibi, A.: Survey: Development and analysis of a gamesbased crisis scenario generation system. In: International Conference on Technologies for E-Learning and Digital Entertainment. pp. 85-100. Springer (2016) 